[Review]

\title{
$\mathrm{CO}$ 加氢制 $\mathrm{C}_{2}$ 含氧化合物 $R h$ 基催化剂中常见助剂的作用
}

\author{
陈维苗 ${ }^{1}$ 丁云杰 ${ }^{1,2, *}$ 薛 飞 ${ }^{1,3}$ 宋宪根 $^{1}$ \\ ('中国科学院大连化学物理研究所, 洁净能源国家实验室(筹), 辽宁大连 116023 ; \\ 2 中国科学院大连化学物理研究所, 催化重点国家实验室, 辽宁大连 116023; ${ }^{3}$ 中国科学院大学, 北京 100049)
}

\begin{abstract}
摘要: 由煤、天然气或生物质出发, 经合成气制乙醇等 $\mathrm{C}_{2}$ 含氧化合物具有重要意义, 负载型 $\mathrm{Rh}$ 基催化剂是实 现该过程最有效的催化剂. 助剂的选择尤其重要, 其中 Fe、Mn、 Li 的助催化作用最为显著, 人们对此进行了长期 而有效的研究, 有关观点也很难统一, 但相关总结性的报道不多. 因此, 本文系统综述了这三种常见助剂催化作 用的研究进展. 结果表明, 这些助剂的作用与其所处的催化体系、制备方法等密切相关, 后者直接影响了助剂金属-载体间相互作用, 使得催化剂各种组分表现出不同存在状态, 进而影响它们在 CO 加氢各基元步骤中的催 化作用. 本文可为人们全面认识这些常用助剂的作用提供有益的参考.
\end{abstract}

关键词: 铑; 合成气； $\mathrm{C}_{2}$ 含氧化合物; 铁; 锰; 锂; 助剂作用; $\mathrm{CO}$ 加氢

中图分类号: 0643

\section{Role of Common Promoters in Rh-Based Catalysts for CO Hydrogenation to $\mathrm{C}_{2}$-Oxygenates}

\author{
CHEN Wei-Miao' DING Yun-Jie ${ }^{1,2, *} \quad$ XUE Fei ${ }^{1,3} \quad$ SONG Xian-Gen ${ }^{1}$ \\ ('Dalian National Laboratory for Clean Energy, Dalian Institute of Chemical Physics, Chinese Academy of Sciences, \\ Dalian 116023, Liaoning Province, P. R. China; ${ }^{2}$ State Key Laboratory of Catalysis, Dalian Institute of Chemical \\ Physics, Chinese Academy of Sciences, Dalian 116023, Liaoning Province, P. R. China; \\ ${ }^{3}$ University of Chinese Academy of Sciences, Beijing 100049, P. R. China)
}

\begin{abstract}
There is great significance to produce $\mathrm{C}_{2}$-oxygenates such as ethanol via syngas from coal, natural gas or biomass from practical and academy of view; and supported Rh-based catalysts are the most effective for this conversion. The choice of promoter is critical for obtaining high-performance catalysts, of which Fe, Mn, and Li are most widely used. The effects of promoters have been studied extensively and comprehensively, but their roles remain the subject of much debate. We review the progress on the promotion nature of $\mathrm{Fe}, \mathrm{Mn}$, and $\mathrm{Li}$. The roles of these promoters depend on the catalyst system and preparation procedures empolyed, which affect directly the interaction between Rh and promoter(s). This interaction determines the displayed structures and properties of the promoters, which behaves different effects on the elemental steps of CO hydrogenation reaction. This review is expected to deeply aid our understanding of the effects of these promoters.
\end{abstract}

Key Words: Rhodium; Syngas; $\mathrm{C}_{2}$-oxygenates; Iron; Manganese; Lithium; Promoter role; CO hydrogenation

\section{1 引 言}

我国缺油少气、人均耕地少, 而煤炭资源相对 丰富, 因此, 从煤、生物质或页岩气出发, 经合成气
直接制取乙醇、乙醛和乙酸等 $\mathrm{C}_{2}$ 含氧化合物具有重 要的战略意义. ${ }^{-21}$ 自 Bhasin 等 ${ }^{4}$ 首次报道了负载型 金属 $\mathrm{Rh}$ 基催化剂上 $\mathrm{CO}$ 加氢反应可高产得到乙醇

Received: September 30, 2014; Revised: November 4, 2014; Published on Web: November 5, 2014.

"Corresponding author. Email: dyj@dicp.ac.cn; Tel/Fax: +86-411-84379143

(C) Editorial office of Acta Physico-Chimica Sinica 
和乙醛等 $\mathrm{C}_{2}$ 含氧化合物以来, 人们在实验上和理论 上对催化剂体系(载体、助剂)和结构性质、反应机 理、制备方法以及构效关系等方面进行了大量而细 致的研究, 然而, 由于 $\mathrm{Rh}$ 具有独一无二的吸附和活 化 $\mathrm{CO}$ 的特性, 因此, 到目前为止, 它仍是实现合成 气制 $\mathrm{C}_{2}$ 含氧化合物最高效的催化活性组分. ${ }^{19-21}$ 本课 题组自上世纪 80 年代以来也对该催化过程进行了 长期和卓有成效的研究, 开发的低含量的 $\mathrm{Rh}$ 基催化 剂正在进行 1 万吨/年的工业放大装置建设. ${ }^{7-18}$

根据普遍接受的 $\mathrm{CO}$ 加氢生成 $\mathrm{C}_{2}$ 含氧化合物的 生成机理 ${ }^{22,23}$ 吸附的 $\mathrm{CO}$ 直接或氢助解离形成烃 类和含氧化合物共同的中间体 $\mathrm{CH}_{x}$ : 它直接加氢 生成甲烷, 或插入 $\mathrm{CO}$ 生成 $\mathrm{C}_{2}$ 含氧化合物的前驱 物 $\mathrm{CH}_{x} \mathrm{CO}$ 、或插入 $\mathrm{CH}_{x}$ 进行链增长; 然后各类中间 体加氢生成相应的烃类和含氧化合物 (见图 1). 可 见, 性能优异的催化剂需保持 $\mathrm{CO}$ 解离和插入活性 之间的较好平衡, 以及适中的加氢活性. 不加助剂 的 $\mathrm{Rh}$ 只是个甲烷化催化剂; 当加入助剂后, 改变了 $\mathrm{Rh}$ 的分散和化学状态, 导致催化剂上进行 $\mathrm{CO}$ 解离、 插入和加氢等基元反应速率发生显著变化, 从而极 大地提高生成 $\mathrm{C}_{2}$ 含氧化合物的活性和选择性. 在 30 多年来所考察的助剂中, Fe、Mn、 Li 和稀土元素的效 果较为突出, 也是研究最多的, 然而相关总结性的 报道不多. 因此, 本文较为系统地总结了 $\mathrm{Fe} 、 \mathrm{Mn} 、 \mathrm{Li}$ 的助催化作用本质, 为 $\mathrm{Rh}$ 基催化剂选择合适助剂提 供一些有益的参考.

\section{2 常见助剂的作用}

\subsection{Fe 助剂}

Bahasin 等 ${ }^{24}$ 报道了在 $2.5 \% \mathrm{Rh}-0.05 \% \mathrm{Fe} / \mathrm{SiO}_{2}$ 催 化剂上 $\mathrm{CO}$ 加氢可生成以乙醇、甲醇和甲烷为主的 产物. 自此, $\mathrm{Fe}$ 对 $\mathrm{Rh}$ 基催化剂的助催化作用的研究 一直延续至今, 是研究最多, 也是合成气直接制乙 醇催化剂中最有效的的助剂之一. 甚至有人断言, 完全无 $\mathrm{Fe}$ 的 $\mathrm{Rh}$ 催化剂上也许没有乙醇的生成. ${ }^{25}$

一般而言, 仅少量的 $\mathrm{Fe}$ 就可显著提高 $\mathrm{Rh}$ 基催

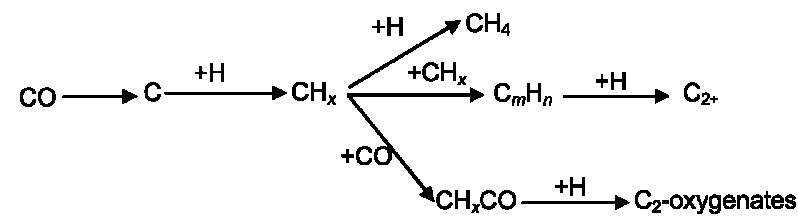

图 $1 \mathrm{Rh}$ 基催化剂上 $\mathrm{CO}$ 加氢的主要反应路径 ${ }^{9}$

Fig.1 Main reaction pathways of $\mathrm{CO}$ hydrogenation over Rh-based catalysts ${ }^{9}$
化剂上 $\mathrm{CO}$ 加氢生成 $\mathrm{C}_{2}$ 含氧化合物主要是乙醇的生 成活性, 同时甲醇的生成活性也随着 $\mathrm{Fe}$ 含量的增加 而显著增加. ${ }^{26-32}$ 研究表明, $\mathrm{Fe}$ 可与 $\mathrm{Rh}$ 形成簇合物或 合金而起着集团效应, ${ }^{26}$ 或覆盖了 $\mathrm{Rh}$ 表面, 使得 $\mathrm{CO}$ 吸附能力下降, 33 特别是多位桥式吸附的 $\mathrm{CO}$, 可导 致 $\mathrm{CO}$ 解离活性下降, ${ }^{27}$ 但 $\mathrm{Fe}$ 通过与 $\mathrm{Rh}$ 的相互作用 在界面处形成了新的吸附和活化 $\mathrm{CO}$ 的活性位, 大 大促进了 $\mathrm{CO}$ 的插入, 使得 $\mathrm{C}_{2}$ 含氧化合物生成活性 增加. ${ }^{34-36} \mathrm{Fe}$ 另外一大作用是显著提高了催化剂的 加氢活性, 导致乙醛和乙酸选择性下降, 乙醇和 $\mathrm{CO}$ 直接加氢生成甲醇的选择性显著增加. $\mathrm{Rh}$ 上活化的 氢可溢流到 $\mathrm{FeO}_{x}$ 上储存起来, 该溢流氢既可使吸附 在孤立 $\mathrm{FeO}_{x}$ 上的乙醛加氢生成乙醇, 成为乙醇生成 路径之一(因为 $\mathrm{Rh} / \mathrm{SiO}_{2}$ 和 $\mathrm{Fe} / \mathrm{SiO}_{2}$ 物理混合的样品 上可使乙醛加氢生成乙醇, 而单独的 $\mathrm{Rh} / \mathrm{SiO}_{2}$ 则不 行 $\left.{ }^{31}\right)$, 同时也可反溢流到 $\mathrm{Rh}-\mathrm{FeO}_{x}$ 活性位处增加其 周围氢浓度, 有利于 $\mathrm{C}_{2}$ 中间物的加氢.

除此之外, $\mathrm{Fe}$ 可在反应过程中稳定 $\mathrm{CH}_{x} \mathrm{CO}$ 基 团, 也有利于其最终转化为乙醇, ${ }^{27}$ 在反应条件下保 持氧化态而有利于含氧化合物的生成; ${ }^{34}$ 改变 $R h$ 催 化剂的酸性, 明显降低甲烷生成速率; ${ }^{37}$ 或稳定了吸 附与 $\mathrm{Rh}^{0}$ 和 $\mathrm{Rh}^{+}$上的 $\mathrm{CO} .^{38}$ 密度泛函理论(DFT) 研究 也表明, ${ }^{39}$ 在 $\mathrm{Rh}(111)$ 中加入 $\mathrm{Fe}$ 使得 $\mathrm{Rh}$ 的 $d$ 能带中心 向 Fermi 能级靠近了 $0.20 \mathrm{eV}$, 从而导致催化剂表面 的 $\mathrm{CH}_{3}$ 和 $\mathrm{H}$ 物种更加稳定, 使得甲烷的生成能垒增 加而受到抑制, 最终导致乙醇生成产率和选择性增 加.

$\mathrm{Fe}$ 的作用取决于它在催化剂中的存在状态与 位置, 而这又与它和 $\mathrm{Rh}$ 及其载体的相互作用密切 相关. Niemantsverdriet等 ${ }^{40,41}$ 采用 Mossbauer 谱研究 了 $3.24 \% \mathrm{Rh}-1.76 \% \mathrm{Fe} / \mathrm{SiO}_{2}(\mathrm{Fe} / \mathrm{Rh}$ 摩尔比为 1$)$ 催化 剂中 $\mathrm{Fe}$ 的存在状态, 发现在 $452{ }^{\circ} \mathrm{C}$ 用 $\mathrm{H}_{2}$ 还原后, $80 \%$ 的 $\mathrm{Fe}$ 物种以 $\mathrm{Fe}^{3+}$ 形式存在, $20 \% \mathrm{Fe}$ 物种为 $\mathrm{Fe}^{0}$, 与至少 1.5 倍的 $\mathrm{Rh}$ 形成合金, $\mathrm{Rh}$ 促进了 $\mathrm{Fe}$ 的还 原. Ichikawa ${ }^{35,36}$ 和 Boffa $\mathrm{A}^{37}$ 等则采用扩展的 $\mathrm{X}$ 射线吸 收精细结构光谱和 Mossbauer 谱研究了金属总载量 为 $0.5 \%$ 的 $\mathrm{Rh}-\mathrm{Fe} / \mathrm{SiO}_{2}$ 体系, 发现随着 $\mathrm{Fe} / \mathrm{Rh}$ 摩尔比 从 0.2 增至 0.5 , 催化剂中 $\mathrm{Fe}^{3+} / \mathrm{Fe}^{0}$ 比从 $88 / 12$ 降至 $73 /$ $27, \mathrm{Fe}^{3+}$ 位于金属-载体界面与 $\mathrm{Rh}$ 原子和载体上 $\mathrm{O}$ 原 子形成化学键, 少量存在的 $\mathrm{Fe}^{0}$ 位于金属 $\mathrm{Rh}$ 的表面. 据此他们提出 $\mathrm{CO}$ 加氢生成 $\mathrm{C}_{2}$ 含氧化合物的活性 位, 即 $\mathrm{Rh}^{0}$ 簇合物与 $\mathrm{Fe}^{3+}$ 相互作用, 形成 $\mathrm{Rh}-\mathrm{Fe}^{3+}-\mathrm{O}$ (载 体)的活性位结构, 可双位活化 $\mathrm{CO}$, 促进了 $\mathrm{CO}$ 的迁 
移与插入, 使得 $\mathrm{CO}$ 加氢 $\mathrm{C}_{2}$ 含氧化合物生成收率显 著增加; 同时还起着针合 $\mathrm{Rh}$ 的作用, 使得 $\mathrm{Rh}$ 稳定地 存在于载体表面. 由此可见, $\mathrm{Rh}-\mathrm{Fe}$ 的界面是活性中 心, 界面面积的控制就显得尤为重要.

载体不同, 其负载的 $\mathrm{Rh}-\mathrm{Fe}$ 催化剂中 $\mathrm{Fe}$ 的状态 也有所不同. 对于 $1 \% \mathrm{Rh}-\mathrm{Fe} / \mathrm{ZrO}_{2}$ 催化剂, ${ }^{34} \mathrm{Rh}-\mathrm{Fe}$ 紧 密接触形成 $\mathrm{Fe}^{x+}$, 起弱 Lewis 酸的作用, 可双位吸附 和活化 $\mathrm{CO}$, 即 Rh-CO-Fe $\mathrm{Fe}^{x+}$, 与 Ichikawa ${ }^{35,36}$ 和 Boffa ${ }^{37}$ 等的类似, 只不过此处 $\mathrm{Fe}$ 物种不仅仅是 $\mathrm{Fe}^{3+}$. 该 $\mathrm{Rh}$ $\mathrm{CO}-\mathrm{Fe}^{x+}$ 既可解离为 $\mathrm{Rh}-\mathrm{C}$ 和 $\mathrm{Fe}-\mathrm{O}$, 也可插入一个 $\mathrm{H}$ 或烷基形成乙酰基物种. 该 $\mathrm{Fe}^{\mathrm{x}}$ 位于金属 $\mathrm{Rh}$ 临近, 在反应条件下可还原为 $\mathrm{Fe}^{0}$. Schunemann 等 ${ }^{42,43}$ 发 现, NaY 分子篮负载的 $2.9 \% \mathrm{Rh}-1.94 \% \mathrm{Fe}$ 催化剂中 $\mathrm{Fe}$ 以 $\mathrm{Fe}^{0}$ 和 $\mathrm{Fe}^{2+}$ 形式存在, 其中 $\mathrm{Fe}^{0}$ 可与 $\mathrm{Rh}^{0}$ 形成合 金, 从而提高催化剂活性; 而 $\mathrm{Fe}^{2+}$ 与 $\mathrm{Rh}$ 颗粒紧密接 触, 作为一个单独氧化物相, 可提高 $\mathrm{CO}$ 加氢生成 $\mathrm{C}_{2}$ 含氧化合物的选择性. 由于该催化剂中 $\mathrm{Rh}$ 和 $\mathrm{Fe}$ 含 量相对较高, 因此在反应条件下 $\mathrm{Fe}^{0}$ 的含量较高, 很 容易与 $\mathrm{Rh}$ 形成合金. 因此,与上述研究结果不太一 致. 对于 $300{ }^{\circ} \mathrm{C}$ 还原后的 $2 \% \mathrm{Rh}-2.5 \% \mathrm{Fe} / \mathrm{TiO}_{2}$ 催化剂 中 $\mathrm{Fe}$ 只能从 +3 还原到 $+2{ }^{4}$ 与之类似, $\mathrm{Al}_{2} \mathrm{O}_{3}$ 负载的 $5 \% \mathrm{Rh}-\mathrm{Fe}$ 催化剂未还原时 $\mathrm{Fe}$ 主要以 $\mathrm{Fe}^{3+}$ 为主, 少量 $\mathrm{Fe}^{2+} ; 500{ }^{\circ} \mathrm{C}$ 还原后大都为 $\mathrm{Fe}^{2+}$, 少量 $\mathrm{Fe}^{3+}$, 且 $\mathrm{Fe}$ 含量 越高, $\mathrm{Fe}^{2+}$ 比例越高. ${ }^{4}$ 总之, $\mathrm{Fe}$ 的状态不仅与载体本 身性质、 $\mathrm{Fe}$ 含量、 $\mathrm{Fe} / \mathrm{Rh}$ 比有关, 也应与还原的温度 与气氛有关; 不同状态和位置的 $\mathrm{Fe}$ 物种起着不同的 作用, 从而影响 $\mathrm{CO}$ 解离、插入和加氢等基元步骤的 进行, 乃至最终 $\mathrm{CO}$ 加氢反应性能.

在 $\mathrm{Rh}-\mathrm{Fe}$ 双组分催化体系中, $\mathrm{Fe}$ 与 $\mathrm{Rh}$ 紧密接触 形成有利于 $\mathrm{C}_{2}$ 含氧化合物形成的活性位, 因此, 提 高 Rh-Fe紧密接触所形成的界面面积则可增加 Rh$\mathrm{Fe}^{3+}-\mathrm{O}$ 活性位数量, 从而提高催化剂性能. ${ }^{32-46}$ 可以 利用载体(如 $\mathrm{Al}_{2} \mathrm{O}_{3}, \mathrm{TiO}_{2}$ 等)较高的活性表面羟基密 度, 形成负载金属氧化物的单覆盖层, 可优化金属 $\mathrm{Rh}$ 与助剂金属氧化物之间的相互作用和接触面积, 从而提高催化剂性能. ${ }^{46}$ 如图 2 所示, 对于 $\mathrm{Rh}-\mathrm{Fe} /$ $\mathrm{Al}_{2} \mathrm{O}_{3}$ 催化剂, 当 $\mathrm{Fe}$ 含量超过载体单层分散容量时 $(10 \%), \mathrm{Rh}-\mathrm{FeO}_{x}$ 界面接触面积开始下降, 乙醇等含 氧化合物生成活性随之下降, 而甲烷选择性上升. 当 $\mathrm{Fe}$ 含量过高, 可单独形成大颗粒的 $\mathrm{Fe}^{0}$ 活性位, 成 为费托反应的活性中心, 使得高碳烃类选择性逐渐 上升. ${ }^{47}$ 因此, 要想得到最大的 $\mathrm{Rh}-\mathrm{FeO}_{x}$ 界面积, 必须 优化 $\mathrm{Fe}$ 含量. 适宜的 $\mathrm{Fe}$ 载量与载体性质(如表面活

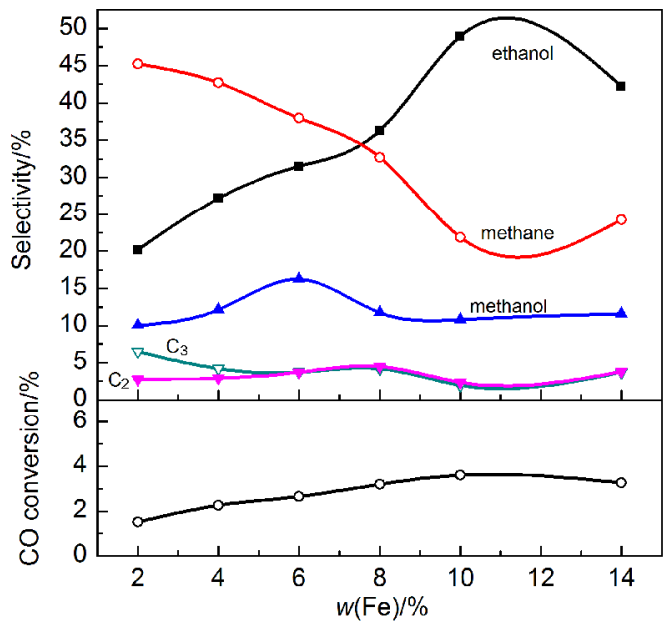

图 $2 \mathrm{Fe}$ 含量对 $\mathrm{Rh}-\mathrm{Fe} / \mathrm{Al}_{2} \mathrm{O}_{3}$ 催化剂上 $\mathrm{CO}$ 加氢 反应性能的影响 ${ }^{46}$

Fig.2 Effect of Fe loading on CO hydrogenation performance over $\mathrm{Rh}-\mathrm{Fe} / \mathrm{Al}_{2} \mathrm{O}_{3}$ catalysts $^{46}$ reaction conditions: $270{ }^{\circ} \mathrm{C}, 1.0 \mathrm{MPa}, n\left(\mathrm{H}_{2}\right) / n(\mathrm{CO})=2,133 \mathrm{~mL} \cdot \mathrm{g}^{-1} \cdot \mathrm{h}^{-1}$

性、比表面积等)密切相关. 对于 $\mathrm{SiO}_{2} 、{ }^{38} \mathrm{SBA}-15 、{ }^{48}$ $\mathrm{ZrO}_{2} 、{ }^{34} \mathrm{Al}_{2} \mathrm{O}_{3}{ }^{46}$ 和 $\mathrm{TiO}_{2}{ }^{38}$ 负载的 $\mathrm{Rh}-\mathrm{Fe}$ 催化剂, 催化剂 性能最佳时的 $\mathrm{Fe} / \mathrm{Rh}$ 摩尔比分别为 $0.9 、 0.925 、 1.5$ 、 2.7 和 4.6. 可以看出, 载体表面活性高, 则适宜的 $\mathrm{Fe} /$ $\mathrm{Rh}$ 比高. 另外, 适宜 $\mathrm{Fe} / \mathrm{Rh}$ 比还与 $\mathrm{FeO}_{x}$ 的引入方式 有关. 当先采用溶胶-凝胶法制得 $\mathrm{FeO}_{x}-\mathrm{SiO}_{2}$, 再浸渍 $\mathrm{Rh}$ 制得催化剂的性能明显好于共浸渍法和一步溶 胶-凝胶法. ${ }^{49}$ 其主要原因就是前者中 $\mathrm{Rh}$ 与 $\mathrm{FeO}_{x}$ 之间 的界面更大, 但此时适宜 $\mathrm{Fe} / \mathrm{Rh}$ 比为 1.35 , 与浸渍法 的 0.27 明显不同.

上述研究仅限于 Rh-Fe 双组分体系, 但对于多 组分体系, 微量 $\mathrm{Fe}$ 的添加就可明显改变催化剂性 能, 所起的作用也不同. 对于 Rh- Mn- $\mathrm{Li} / \mathrm{SiO}_{2}$ 催化 剂, ${ }^{8,50-52}$ 当 $\mathrm{Fe}$ 含量很低时 $(0.01 \%-0.1 \%), \mathrm{Fe}$ 物种主 要以 $\mathrm{Fe}$ 氧化物的形式高度分散在载体表面, 部分 $\mathrm{Rh}$ 物种针定在 $\mathrm{Fe}$ 氧化物表面而形成 $\mathrm{Rh}-\mathrm{FeO}_{x}-\mathrm{SiO}_{2}$ 夹心结构, 组分之间紧密接触, $\mathrm{C}_{2}$ 含氧化合物的生 成活性和选择性增加, 其中乙醛选择性上升, 而乙 醇选择性反而下降, 与 Rh-Fe 双组分催化剂体系有 所不同, 这可能与 $\mathrm{Fe}$ 含量非常低而高度分散, 且与 $\mathrm{Rh}$ 紧密接触, 单独形成乙醛加氢活性位的部分很少 有关. 当 $\mathrm{Fe}$ 含量超过 $0.05 \%$ 时, 则位于 $\mathrm{Rh}$ 附近的 $\mathrm{Fe}$ 物种形成乙醛加氢活性位, 使得乙醇和甲醇选择性 明显上升,而乙醛选择性下降; 至 $0.1 \%$ 时催化剂活 性开始显著下降. 傅里叶变换红外(FT-IR)光谱结果 显示, ${ }^{51}$ 不同含量的 $\mathrm{Fe}$ 对吸附 $\mathrm{CO}$ 的脱附/转化行为 的影响不同, 随着 $\mathrm{Fe}$ 含量增加, $\mathrm{Fe}$ 覆盖了 $\mathrm{Rh}$ 而使得 
催化剂上 $\mathrm{CO}$ 吸附量下降, 但孪式 $\mathrm{CO}$ 的脱附/转化 速率增加占主导作用, 因而催化剂性能改善; 当 $\mathrm{Fe}$ 含量超过 $0.1 \%$ 时, $\mathrm{CO}$ 吸附量的下降占主导作用, 因 而催化剂活性下降, 而 $\mathrm{C}_{2}$ 含氧化合物选择性的下降 则归因于孪式吸附 $\mathrm{CO}$ 转化为 $\mathrm{H}-\mathrm{Rh}-\mathrm{CO}$.

$\mathrm{Mo}$ 等 ${ }^{53}$ 也对多组分的 $1.5 \% \mathrm{Rh}-\mathrm{La}-\mathrm{V}-\mathrm{Fe} / \mathrm{SiO}_{2}$ 催 化剂中各助剂作用进行了研究. 结果表明, $\mathrm{Fe}$ 的添 加抑制了 $\mathrm{CO}$ 吸附, 但在反应温度时 $\mathrm{H}_{2}$ 的吸附量更 高, 提高了催化剂加氢能力, 尤其是 $\mathrm{C}=\mathrm{O}$ 的加氢, 因而乙醇选择性更高; 但却不一定促进 $\mathrm{C}=\mathrm{C}$ 的加 氢, 因此烯烷比不一定很低. 需要指出的是, 此处 $\mathrm{Fe}$ 含量相对较高, 为 $0.8 \%$, 因此其表现出的作用也与 $\mathrm{Yin}^{8}$ 和 $\mathrm{Yu}^{50}$ 等的不同. 总之, 在多组分促进的 $\mathrm{Rh}$ 基 催化剂中, $\mathrm{Fe}$ 所表现出来的作用与其含量更是密切 相关.

\section{$2.2 \mathrm{Mn}$ 助剂}

与 $\mathrm{Fe}$ 可能会降低催化剂的 $\mathrm{CO}$ 解离活性不同, $\mathrm{Mn}$ 的加入则显著提高了 $\mathrm{Rh}$ 上 $\mathrm{CO}$ 解离活性, 且稳 定了表面 $\mathrm{C}_{2}$ 含氧化合物中间体, 从而显著提高 $\mathrm{Rh}$ 基催化剂的活性和生成 $\mathrm{C}_{2}$ 含氧化合物的选择性; ${ }^{26}$ 但也与 $\mathrm{Fe}$ 一样, 以乙醛为代价而促进乙醇的生 成, 只是不及 $\mathrm{Fe}$ 明显, 它主要是促进乙酸的生成. ${ }^{29}$ Sachtler 等 ${ }^{4}$ 根据吸附于 $\mathrm{Rh}$ 上 $\mathrm{CO}$ 伸缩频率大大降 低的现象, 同时受均相体系中 L 酸可配位于配合物 中 $\mathrm{CO}$ 的 $\mathrm{O}$ 端的启发, ${ }^{55}$ 提出了 $\mathrm{Mn}$ 等亲氧性助剂促 进的 $\mathrm{Rh}$ 基催化剂上 $\mathrm{CO}$ 活化的新模式, 即形成了一 种倾斜式吸附的 $\mathrm{CO}$ 物种, 如图 3 所示, $\mathrm{CO}$ 的 $\mathrm{C}$ 端吸 附在金属 $\mathrm{Rh}$ 上, $\mathrm{O}$ 端倾斜指向 $\mathrm{Mn}$ 阳离子, 这类似于 均相中 $\mathrm{L}$ 酸碱的作用, 大大削弱了 $\mathrm{C}-\mathrm{O}$ 键, 从而促 进了 $\mathrm{CO}$ 的解离, 因而催化活性大大提高.

Wilson 等 ${ }^{56}$ 用顺磁共振光谱(EPR) 研究了 Rh$\mathrm{Mn} / \mathrm{SiO}_{2}$ 催化剂中 $\mathrm{Mn}$ 的助催化作用, 他们设想 $\mathrm{MnO}$ 与 $\mathrm{Rh}$ 物种形成了 $\mathrm{Rh}-\mathrm{O}-\mathrm{Mn}$ 复合氧化物, 从而降低 了 $\mathrm{CO}$ 的解离速率, 减少了表面积碳, 提高了金属的
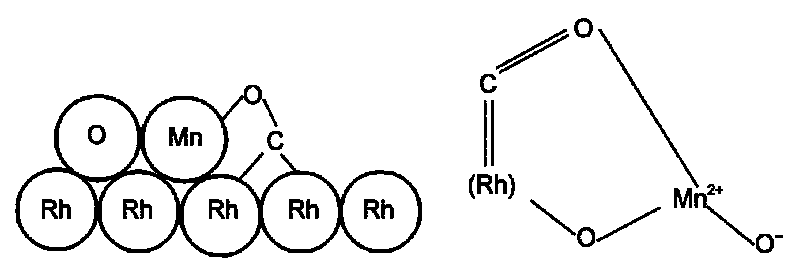

图 3 亲氧性 $\mathrm{Mn}$ 助剂存在下 $\mathrm{CO}$ 在 $\mathrm{Rh}$ 基 催化剂上的化学吸附模型

Fig.3 Titled CO chemisorptions modes on $\mathrm{Rh}$ with the existence of oxyphilic Mn promoter
有效表面积, 使得催化剂活性增加. Rh-O-Mn 复合 氧化物的形成也使得 $\mathrm{Rh}$ 很难被还原, 可能减弱了 $\mathrm{CO}$ 的吸附, 从而增加表面 $\mathrm{H}$ 还原的浓度, 使得活性 增加; 5 同时催化剂表面的 $\mathrm{Rh}^{+}$物种则会增加, 有可 能抑制了 $\mathrm{Rh}$ 和 $\mathrm{Mn}$ 组分在载体表面的移动和聚集, 从而提高了催化剂上 $\mathrm{Rh}$ 分散度, 也使得 $\mathrm{C}_{2}$ 含氧化 合物生成活性增加; $;^{58,59}$ 或形成了新的 $\mathrm{CO}$ 插入活性 位 $\left(\mathrm{Rh}_{x}^{0} \mathrm{Rh}_{y}^{+}\right)-\mathrm{O}-\mathrm{Mn}^{2+}(x>>y){ }^{60} \mathrm{Ojeda}$ 等 ${ }^{61}$ 也认为, 在 $\mathrm{Mn}-\mathrm{Rh} / \mathrm{Al}_{2} \mathrm{O}_{3}$ 催化剂中 $\mathrm{MnO}_{x}$ 起到拉电子作用, 部分 氧化 $\mathrm{Rh}-\mathrm{MnO}$ 界面处的 $\mathrm{Rh}$ 原子为 $\mathrm{Rh}^{\delta+}$, 从而形成新 的 $\mathrm{CO}$ 插入活性位, 因而含氧产物, 主要是乙醇的选 择性增加. 这些观点基本认同催化剂中存在氧化态 的 $\mathrm{Rh}$, 但也有人认为, 在反应条件下 $\mathrm{Rh}$ 物种应该很 容易完全还原为金属态, 特别是低转化率时体系中 水汽含量很低的情况下. 因此保持氧化态的助剂离 子则对催化剂选择性的提高起着重要作用. ${ }^{34,53}$

Trevino 等 ${ }^{62}$ 研究了 Rh-Mn/NaY 催化剂中金属一 助剂间相互作用的本质, 却认为是 $\mathrm{MnO}$ 颗粒, 而不 是 $\mathrm{Mn}^{2+}$ 促进了催化剂上 $\mathrm{CO}$ 加氢生成 $\mathrm{C}_{2}$ 含氧化合物 的生成; 催化剂中并不存在金属 $\mathrm{Mn}$ 或 $\mathrm{Rh}-\mathrm{Mn}$ 合金, 因为 $\mathrm{Mn}$ 物种还原到金属 $\mathrm{Mn}$ 需 $1000{ }^{\circ} \mathrm{C}$ 高温, 即使 $\mathrm{Rh}$ 的存在促进了 $\mathrm{Mn}$ 物种的还原, 也很难得到金属 $\mathrm{Mn}$. 但 $\mathrm{Rh}$ 的存在大大促进了 $\mathrm{MnO}_{2}$ 还原为 $\mathrm{MnO}$, 表 明 $\mathrm{Rh}$ 簇合物和 $\mathrm{MnO}$ 的紧密接触; $\mathrm{MnO}$ 影响了 $\mathrm{Rh}$ 吸附 $\mathrm{H}_{2}$ 的能力. 该作者还据此提出了合成气制 $\mathrm{C}_{2}$ 含氧化合物的新机理, ${ }^{63}$ 认为含氧化合物的前驱体 $\mathrm{C}_{x} \mathrm{H}_{y} \mathrm{O}_{z}$ 在于 $\mathrm{Rh}-\mathrm{MnO}$ 临近的 $\mathrm{MnO}$ 上形成, 而 $\mathrm{Rh}$ 的作 用是形成并传输 $\mathrm{CH}_{x}$ 物种和 $\mathrm{H}$ 原子, 如图 4 所示, 助 剂 $\mathrm{MnO}$ 通过稳定 $\mathrm{Rh}$ 粒子上很容易分解的表面乙酸 根物种来提高催化剂选择性.

上述观点基本都认为不会形成金属 $M n$ 或 $R h-$

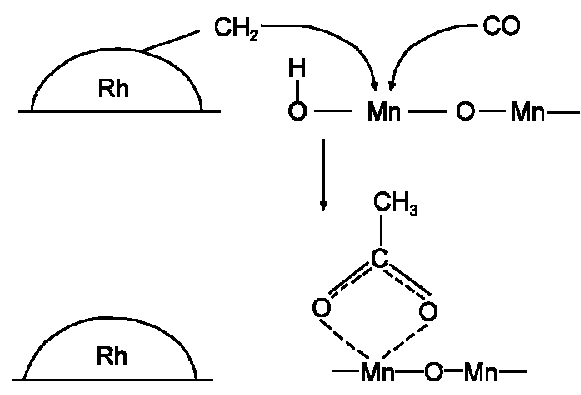

图 4 临近 $\mathrm{Rh}$ 粒子的 $\mathrm{MnO}$ 位上 $\mathrm{CH}_{2} 、 \mathrm{CO}$ 和表面 羟基之间的相互作用 ${ }^{63}$

Fig.4 Interaction between $\mathrm{CH}_{2}, \mathrm{CO}$ and surface hydroxyl on MnO sites near the Rh particle ${ }^{63}$ 
$M n$ 合金, ${ }^{53-64}$ 然而, Mei 等 ${ }^{65}$ 结合实验和基于第一性 原理的动力学模拟研究了硅胶负载的 $\mathrm{Rh} / \mathrm{Mn}$ 合金 催化剂上 $\mathrm{CO}$ 加氢生成乙醇的反应. $\mathrm{X}$ 射线光电子 能谱(XPS), 透射电镜(TEM) 和 X射线衍射(XRD)等 表征结果都证实了催化剂中形成了 $\mathrm{Rh} / \mathrm{Mn}$ 合金, 是 $\mathrm{C}_{2}$ 含氧化合物的生成活性位. 理论计算结果也表 明, 在还原性的反应气氛和较高的温度下, $\mathrm{Rh} / \mathrm{Mn}$ 二元合金的热力学稳定性要高于 $\mathrm{Rh}-\mathrm{MnO}_{x}$ 混合氧 化物; 形成的 Rh-Mn二元合金不影响甲烷生成能 垒, 且 $\mathrm{CO}$ 插入 $\mathrm{CH}_{2}$ 和 $\mathrm{CH}_{3}$ 的反应能垒仍较高, 但却 降低了 $\mathrm{CO}$ 插入 $\mathrm{CH}$ 反应能垒, 从而使得乙醇等 $\mathrm{C}_{2}$ 含 氧化合物的选择性增加. 理论结果和实验结果吻合 得较好. 作者进一步指出, 由于表征时样品接触到 空气中氧, Rh-Mn 合金很容易被氧化, 因而很难被 观察到. 由此可见催化剂原位表征的重要性.

另外, 人们还在理论上揭示了 $\mathrm{Mn}$ 对 $\mathrm{Rh}$ 助催化 作用的本质. Ma 等 ${ }^{66}$ 采用 DFT 比较了 $\mathrm{Mn}$ 的加入对 $\mathrm{Rh}$ (111)和 $\mathrm{Rh}$ (553)面上 $\mathrm{CO}$ 吸附和解离性能的影响, 发现 $\mathrm{C}-\mathrm{O}$ 键断裂能垒取决于产物生成能垒, 且两 者存在良好的线性关系. 与 $\mathrm{Rh}(111)$ 面相比, $\mathrm{Mn}$ 的加 入使得 $\mathrm{Rh}(553)$ 上 $\mathrm{CO}$ 解离能垒下降了 $1.60 \mathrm{eV}$. 另 外, $\mathrm{Mn}$ 和 $\mathrm{Rh}$ 阶梯面的存在可明显促进 $\mathrm{CO}$ 的解离. $\mathrm{Li}$ 等 ${ }^{67}$ 则认为, 当金属态的 $\mathrm{Mn}$ 覆盖在 $\mathrm{Rh}$ 表面时, $\mathrm{CO}$ 解离和插入能垒均下降.

如上所述, $\mathrm{Mn}$ 与 $\mathrm{Rh}$ 之间的紧密接触是提高催 化剂催化 $\mathrm{CO}$ 加氢生成 $\mathrm{C}_{2}$ 含氧化合物的关键, 因此, 如何选择和改进催化剂制备方法显得尤为重要. Huang 等 ${ }^{68}$ 以硅酸乙酯和十六烷基三甲基溴化铵为 原料, 在原位生成中孔硅胶载体的体系中加入 $\mathrm{Rh}$ 纳 米粒子和 $\mathrm{Mn}\left(\mathrm{NO}_{3}\right)_{2}$, 使得 $\mathrm{Mn}$ 粒子和 $\mathrm{Rh}$ 粒子周围均 匀分布, 且接触更为紧密, 所制催化剂在 $\mathrm{CO}$ 加氢反 应生成 $\mathrm{C}_{2}$ 含氧化合物的选择性高达 $74.5 \%$. Liu 等 ${ }^{69}$ 利用载体和 $\mathrm{Rh}_{2} \mathrm{O}_{3}$ 的等电点的差异, 通过控制浸渍 液 $\mathrm{pH}$ 值采用强静电吸附(SEA) 法将 $\mathrm{MnO}_{4}^{-}$选择吸附 到已预先负载在 $\mathrm{SiO}_{2}$ 上 $\mathrm{Rh}_{2} \mathrm{O}_{3}$ 的表面, 从而制得 $3 \%$ $\mathrm{Rh}-\mathrm{Mn} / \mathrm{SiO}_{2}$. 结果发现, 采用 SEA 法制得催化剂还 原后 Rh-Mn 间相互作用的程度高于普通浸渍法制 得的, 且对 $\mathrm{Rh}$ 物种的还原性能的影响很小, 但在 $\mathrm{CO}$ 加氢反应中乙醇选择性更高. 这种选择吸附法也可 用于制备其它载体和助剂中, 以得到理想的金属-载 体相互作用.

$\mathrm{Liu}$ 等 ${ }^{70}$ 还发现, 通过改变 Mn 含量可调节 Rh$\mathrm{Mn}$ 间相互作用的大小以提高多壁碳纳米管
(MCNTs) 负载的 $3 \% \mathrm{Rh}-\mathrm{Mn} /$ 催化剂上 $\mathrm{CO}$ 加氢生成 乙醇的活性和选择性. 当 $\mathrm{Mn}$ 含量从 $1 \%$ 增至 $2 \%$ 时, 表征结果证实, 尽管与不加 $\mathrm{Mn}$ 的催化剂一样, $\mathrm{Rh}$ 粒 径仍为 $1 \mathrm{~nm}$, 但 $\mathrm{Rh}-\mathrm{Mn}$ 间相互作用增强, 使得乙醇 选择性增加. 另有研究发现, 适量 $\mathrm{Li}^{71}$ 或微量 $\mathrm{Ti}^{10,11}$ 的 加入也可调节 $\mathrm{Rh}-\mathrm{Mn}$ 相互作用的程度, 从而改变 催化剂性能. 总体上, Rh-Mn相互作用程度适中的 催化剂性能较高. 然而, 最近的报道显示, ${ }^{72} \mathrm{MCNTs}$ 负载的 $5 \% \mathrm{Rh}-5 \% \mathrm{Mn}$ 催化剂中, $\mathrm{Mn}$ 物种以 $\mathrm{MnO}$ 形 式存在, 且与 $\mathrm{Rh}$ 无明显的相互作用, 遗憾的是, 作者 并未提供相应的 CO 加氢反应结果. 该实验现象值 得深思和进一步研究, 这很可能与该催化剂载体的 特殊性质以及 $\mathrm{Rh} 、 \mathrm{Mn}$ 含量过高有关.

归纳起来, $\mathrm{Mn}$ 通过与 $\mathrm{Rh}$ 的相互作用在界面处 形成了复合氧化物、紧密接触的 $\mathrm{Rh}$ 与 $\mathrm{MnO}$ (或 $\mathrm{Mn}^{2+}$ )、甚至 Rh-Mn 合金等, 提高了 $\mathrm{Rh}$ 的分散度或改 变了 $\mathrm{Rh}$ 的电子性质, 改善了 $\mathrm{CO}$ 吸附或活化模式, 促进了 $\mathrm{CO}$ 解离及其插入, 从而极大提高了催化剂 性能.

\section{3 碱金属助剂}

碱金属是合成气制醇类不可或缺的催化剂助 剂, 但其作用则与 $\mathrm{Fe} 、 \mathrm{Mn}$ 等过渡金属明显不同. $\mathrm{Li}$ 、 $\mathrm{K} 、 \mathrm{Na}$ 对催化剂性能的影响很类似, 其对催化剂活 性影响程度的大小顺序为 $\mathrm{Na}<\mathrm{Li}<\mathrm{K}$. ${ }^{5}$ 但它们对催化 剂选择性的影响也有差异, 如对于 $\mathrm{Rh} / \mathrm{La}_{2} \mathrm{O}_{3}$ 体系, 其 催化 $\mathrm{CO}$ 加氢反应产物主要是甲醇, $\mathrm{Li}$ 的加入可提 高 $\mathrm{C}_{2}$ 含氧化合物选择性, $\mathrm{K}$ 的加入对产物分布没有 影响, 而 $\mathrm{Cs}$ 的加入却抑制了 $\mathrm{C}_{2}$ 含氧化合物的生成. ${ }^{73}$ 另外, 这些碱金属助剂对催化剂的稳定性影响也很 大, 如 $\mathrm{Na}$ 的添加对 $\mathrm{Rh}-\mathrm{Sc} / \mathrm{SiO}_{2}$ 上 $\mathrm{CO}$ 加氢生成甲烷 和高碳化合物生成的稳定性影响不大, 但对生成乙 醛和乙酸的影响很大, 当 $\mathrm{Na} / \mathrm{Rh}$ 原子比小于 0.14 时, 催化剂稳定性较高.

在许多催化过程中, 催化剂中碱金属主要被当 做电子性助剂, 但有时也起几何性助剂, 覆盖 $\mathrm{Rh}$ 表 面、稀释活性金属表面的作用. Blackmond 等 ${ }^{74}$ 研究 $\mathrm{Cs}$ 对 $\mathrm{Rh} / \mathrm{Al}_{2} \mathrm{O}_{3}$ 催化剂助催化作用时发现, $\mathrm{Cs}$ 大部分 直接与载体, 而不是 $\mathrm{Rh}$ 晶粒发生相互作用; $\mathrm{Cs}$ 的作 用主要是覆盖了 $\mathrm{Rh}$ 位而抑制了其吸附性能. 当选择 相对惰性的、并具适当物性的 $\mathrm{SiO}_{2}$ 作为载体, 在制 备过程中使得 $\mathrm{K}$ 物种与 $\mathrm{Rh}$ 紧密接触, 发生较强相互 作用, 此时则可起到电子助剂的作用, 否则主要起 几何助剂的作用. ${ }^{73}$ Gallaher 等 ${ }^{75}$ 研究发现, 对于 $\mathrm{Rh} /$ 
$\mathrm{SiO}_{2}$ 体系, $\mathrm{Rb}$ 的加入修饰了 $\mathrm{Rh}$ 表面, 抑制了 $\mathrm{Rh}$ 的 吸附和催化活性, 但并未改变 $\mathrm{Rh}$ 的电子性质. 而对 于 $\mathrm{Rh} / \mathrm{La}_{2} \mathrm{O}_{3}$ 体系, $\mathrm{K}$ 和 $\mathrm{Rb}$ 的加入堵塞了 $\mathrm{Rh}$ 上吸附 $\mathrm{H}$ 溢流到 $\mathrm{LaO}_{x}$ 或载体的活性位或途径, 其催化活性并 未因碱金属对 $\mathrm{Rh}$ 活性位的稀释而受到影响; 同时, $\mathrm{Rh}$ 或 $\mathrm{Rb}$ 的化学态均略有改变. 由此可见, 碱金属所 起的作用与催化剂体系和制备过程密切相关. 对于 $\mathrm{Rh} / \mathrm{TiO}_{2}$ 体系, ${ }^{76}$ 各碱金属对含氧化合物选择性大小 顺序为: 无碱金属 $<\mathrm{Li}<\mathrm{K}=\mathrm{Cs}$, 但 $\mathrm{CO}$ 转化率的大小 顺序为: 无碱金属 $>\mathrm{Li}>\mathrm{K}>\mathrm{Cs}$.

比较而言, $\mathrm{Li}$ 是最常用的碱金属助剂, 它可提 高乙醛和乙酸选择性, 降低烃类, 特别是甲烷选择 性, 但往往会造成 CO 加氢活性的降低. Burch 等 ${ }^{31}$ 研 究发现, 在不加 $\mathrm{Li}$ 的 $\mathrm{SiO}_{2}$ 负载的 $\mathrm{Rh}$ 基催化剂上, 乙 酫和丙烷生成活性之间存在很好的对应关系, 表明 二者来自于共同的中间物种一乙酰基; 但 $\mathrm{Li}$ 的加 入却打破了这种关系, 表明 $\mathrm{Li}_{2} \mathrm{O}$ 位于金属 $\mathrm{Rh}$ 表面, 但可能不能像亲氧性助剂那样储氢, 或抑制了氢的 溢流. ${ }^{75}$ 这表明 $\mathrm{Li}$ 的加入抑制了催化剂的加氢能力, 动力学和 $\mathrm{H}_{2}$-程序升温脱附结果均证实, 碱金属助剂 的加入增大了 $\mathrm{H}_{2}$ 的反应级数 ${ }^{31}$ 或吸附 $\mathrm{H}_{2}$ 的数量急 剧减少. ${ }^{73,74}$ 催化剂加氢能力的下降导致氢助解离 $\mathrm{CO}$ 生成 $\mathrm{CH}_{x}$ 的速率下降, 因而催化剂活性和烃类选 择性降低; 同时也抑制了乙酰基, 甚至乙醛的加氢, 因而有利于 $\mathrm{C}_{2}$ 中间物转化为加氢程度最低的乙酸, 使其选择性上升. $\mathrm{Rh} / \mathrm{TiO}_{2}$ 催化剂具有较高的催化 $\mathrm{CO}$ 加氢生成乙醇反应性能, 但也生成了大量的烃 类. ${ }^{77,78} \mathrm{Li}$ 的添加抑制了 $\mathrm{Rh} / \mathrm{TiO}_{2}$ 催化剂上 $\mathrm{CO}$ 加氢反 应中甲烷和甲醇的生成, 从而进一步提高 $\mathrm{C}_{2}$ 含氧化 合物的生成活性. ${ }^{75}$ 进一步研究发现, ${ }^{79} \mathrm{Li}$ 的添加只是
稍稍降低了 $\mathrm{Rh} / \mathrm{TiO}_{2}$ 催化剂中 $\mathrm{Rh}$ 粒径(见图 5(a)), 使 得 $\mathrm{CO}$ 解离能力下降, 因而活性有所下降; $\mathrm{Li}$ 也未起 到电子助剂的作用, 因为在反应温度下只观察到吸 附在 $\mathrm{Rh}^{0}$ 上的 $\mathrm{CO}$ 吸附物种 (见图 5(b)), 此时催化剂 中 $\mathrm{Rh}$ 基本以 $\mathrm{Rh}^{0}$ 存在(见图 5(c)); 但 $\mathrm{Li}$ 的加入却产 生了一种弱的 $\mathrm{CO}$ 吸附物种, 这可能是导致含氧化 合物选择性升高的原因. Kusama等 ${ }^{80}$ 考察了 30 多种 助剂对 $5 \% \mathrm{Rh} / \mathrm{SiO}_{2}$ 催化剂上 $\mathrm{CO}_{2}$ 加氢制乙醇反应性 能的影响, 也发现 $\mathrm{Li}$ 的效果最好, 作者认为, $\mathrm{Li}$ 增加 了表面 $\mathrm{Rh}$ 的电子密度, 削弱了吸附物种的 $\mathrm{C}-\mathrm{O}$ 键, 从而促进了中间体 $\mathrm{CO}$ 的生成.

一般而言, 单独 $\mathrm{Li}$ 促进的 $\mathrm{Rh}$ 催化剂性能并不 高, 需在多组分的 $\mathrm{Rh}$ 基催化剂中才能有效发挥其催 化作用, 其作用机制也变得复杂. $\mathrm{Ngo}$ 等 ${ }^{81}$ 在 $2 \% \mathrm{Rh}$ $5 \% \mathrm{Fe} / \mathrm{TiO}_{2}$ 催化剂中添加了 $0.25 \% \mathrm{Li}$, 在较低温度和 压力条件下, 虽然 $\mathrm{CO}$ 转化率从 $14 \%$ 降至 $10 \%$, 但大 大降低了烃类选择性, 乙醇的时空收率和选择性显 著增加. Wang 等 ${ }^{71}$ 采用 EPR、XPS、FT-IR 和程序升温 还原等技术考察了 $\mathrm{Li}$ 对 $\mathrm{Rh}-\mathrm{Mn} / \mathrm{SiO}_{2}$ 催化剂的助催 化作用, 认为 $\mathrm{Mn}$ 作为电子受体, 而 $\mathrm{Li}$ 却具有电子给 体效应. Li 的添加可抑制 Rh-Mn 复合氧化物的形 成, 并增加催化剂表面 $\mathrm{Rh}^{0}$ 的浓度; 另外 $\mathrm{Li}$ 还可能导 致 $\mathrm{Rh}$ 上倾斜式吸附 $\mathrm{CO}$ 的形成和 $\mathrm{Rh}$ 上吸附氢溢流 到载体表面. 李经伟等 ${ }^{15}$ 则发现, $\mathrm{Li}$ 的加入减少了 $\mathrm{Rh}-\mathrm{Mn} / \mathrm{SiO}_{2}$ 催化剂上能够解离 $\mathrm{CO}$ 活性位的数量, 但却增加了催化剂上 $\mathrm{CO}$ 插入活性位的数量, 分别 导致催化剂活性降低和 $\mathrm{C}_{2}$ 含氧化合物选择性增加. $\mathrm{Li}$ 对 $\mathrm{Rh}$ 基催化剂上 $\mathrm{H}_{2}$ 和 $\mathrm{CO}$ 吸附量的影响很小, 这 可能是因为有很大一部分 $\mathrm{Li}$ 没有与 $\mathrm{Rh}$ 发生相互作 用, 而是分散到了载体 $\mathrm{SiO}_{2}$ 上, 而少部分 $\mathrm{Li}$ 覆盖在
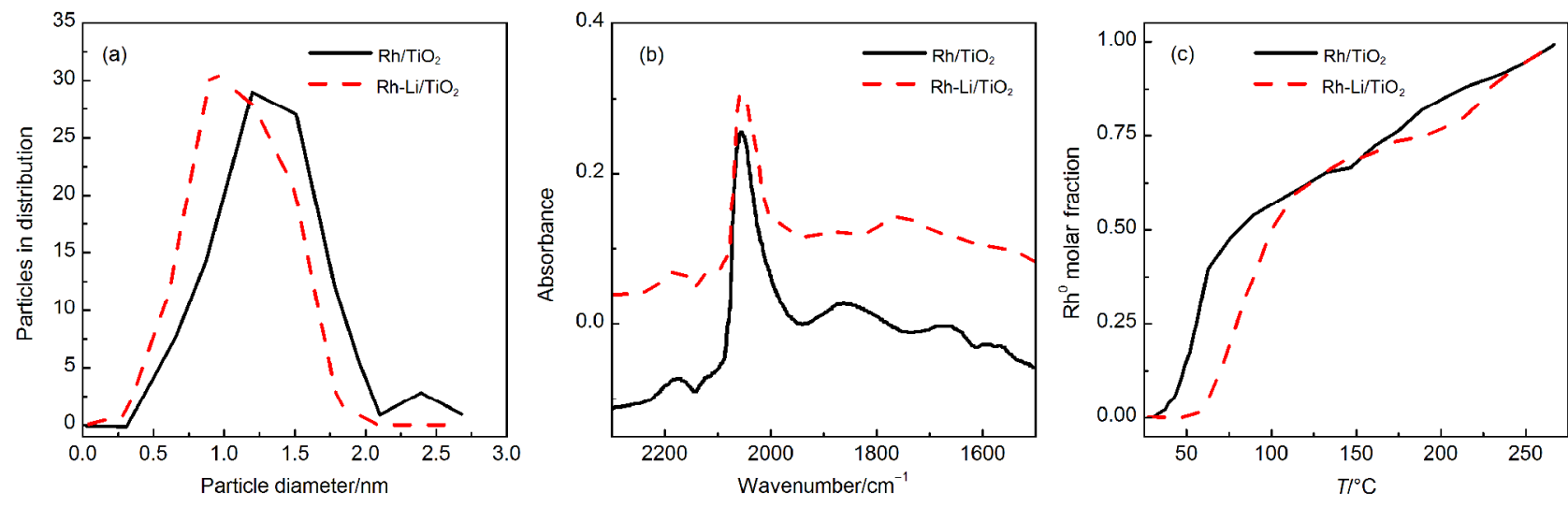

图 $5 \mathrm{Rh} / \mathrm{TiO}_{2}$ 和 $\mathrm{Rh}-\mathrm{Li} / \mathrm{TiO}_{2}$ 催化剂 $\mathrm{Rh}$ 粒径分布图 $(\mathrm{a}){ }^{78}{ }^{70} 270{ }^{\circ} \mathrm{C}$ 吸附 $\mathrm{CO}$ 的 FT-IR 谱图 $(\mathrm{b})^{79}$ 及其 $\mathrm{Rh}^{0}$ 摩尔分数随温度变化图 $(\mathrm{c})^{79}$ Fig.5 Rh particle size distribution (a), ${ }^{78}$ FT-IR spectra for $\mathrm{CO}$ adsorption at $270{ }^{\circ} \mathrm{C}(\mathrm{b}),{ }^{79}$ and $\mathrm{Rh}^{0}$ molar fraction as a function temperature $(c)^{79}$ for $\mathrm{Rh} / \mathrm{TiO}_{2}$ and $\mathrm{Rh}-\mathrm{Li} / \mathrm{TiO}_{2}$ catalysts 
了 $\mathrm{Rh}$ 的表面,使 $\mathrm{Rh}$ 的还原变得困难. 在其它的碱金 属对不同载体负载 $\mathrm{Rh}$ 催化剂性能的影响的研究结 果也表明, ${ }^{71-75}$ 碱金属的落位, 及其与 $\mathrm{Rh}$ 或载体相互 作用的程度影响了催化剂的吸附和催化性能, 而这 些相互作用的程度与载体的性质和催化剂的制备 过程是密切相关的. 这也可能导致 $\mathrm{Wang}^{71}$ 和李经 伟 ${ }^{15}$ 等研究结果不太一致的原因.

综上所述, Li 助催化作用可解释为, 部分覆盖 $\mathrm{Rh}$ 表面, 影响 $\mathrm{Rh}$ 的分散或氢的溢流, 或与 $\mathrm{Rh}$ 紧密 接触起电子性助剂作用, 改变了 $\mathrm{Rh}$ 的化学态, 使得 催化剂加氢和解离 $\mathrm{CO}$ 的能力下降, 抑制烃类的生 成并提高乙醇, 特别是乙酸等 $\mathrm{C}_{2}$ 含氧化合物的选择 性.

与碱金属一样, 碱土金属对 $\mathrm{Rh} / \mathrm{SiO}_{2}$ 催化剂活性 顺序为: $\mathrm{Rh}-\mathrm{Mg} / \mathrm{SiO}_{2}>\mathrm{Rh}-\mathrm{Ca} / \mathrm{SiO}_{2}>\mathrm{Rh}-\mathrm{Sr} / \mathrm{SiO}_{2}$, 与碱 土金属的碱性强弱的顺序一致, 其中 $\mathrm{Rh}-\mathrm{Mg} / \mathrm{SiO}_{2}$ 催 化剂活性高于 $\mathrm{Rh}-\mathrm{Li} / \mathrm{SiO}_{2}$, 但生成 $\mathrm{C}_{2}$ 含氧化合物选 择性更低.一般而言, 碱金属的促进效果好于碱土 金属的. 另外, 胺等有机碱或碱土金属的加入也可 提高 $\mathrm{Rh}-\mathrm{Fe} / \mathrm{Al}_{2} \mathrm{O}_{3}$ 催化剂上甲醇同系化反应制乙醇 和乙酸甲酯反应性能, 82 但在 $\mathrm{CO}$ 加氢反应中, 其促 进作用不大. ${ }^{17}$ 另一方面, Li、Na、K 和 Cs 等碱金属和 $\mathrm{Sr} 、 \mathrm{Ba}$ 等碱土金属助剂可中和催化剂表面酸性, 从 而抑制了异构化、脱水和积碳等副反应的发生; 少 量的添加通常会提高催化剂活性, 但过高的载量会 堵塞催化剂表面活性位使比表面积下降而失活.

\section{3 助剂金属电负性和酸碱性与其催化作用 的关联}

Mei 等 ${ }^{65}$ 运用 DFT 计算将各类助剂 $M$ 和 $\mathrm{Rh}$ 金 属电负性的差值 $\Delta \chi$ 与 $\mathrm{CO}$ 插入基元反应的能垒进行 了关联, 计算时假定位于以 $\mathrm{Rh}$ 为主的簇合物 $\left(\mathrm{Rh}_{49} \mathrm{M}_{1}\right.$, 粒径约 $\left.1 \mathrm{~nm}\right)$ 中的助剂 $\mathrm{M}$ 在反应时被还原 到金属态(实际上有些助剂很难还原到金属态, 尽管 其周围大量 $\mathrm{Rh}$ 有利于助剂氧化物的还原). 如图 6 所示, $\mathrm{CO}$ 插入到 $\mathrm{CH}$ 反应的活化能垒随着 $\Delta \chi$ 接近 0.70 而逐渐降低, 然后又随着 $\Delta \chi$ 继续增加而上升; 其中将 $\mathrm{Ti}$ 与 $\mathrm{Rh}$ 组成合金时, 该能垒最低, 预示着 $\mathrm{Ti}$ 可能是 $\mathrm{Rh}$ 催化剂中较为适宜的助剂, 可显著提高 $\mathrm{CO}$ 加氢反应生成的乙醇等 $\mathrm{C}_{2}$ 含氧化合物选择性和 活性. 当 $\Delta \chi$ 接近 0.70 时, $\mathrm{CO}$ 插入活性形成的反应物 种过渡态可稳定地存在于助剂位 $\mathrm{M}^{\delta+}$ 上, 后者作为 Lewis 酸位, 而 $\mathrm{Rh}^{\delta-}$ 作为 Lewis 碱位使得反应物分子

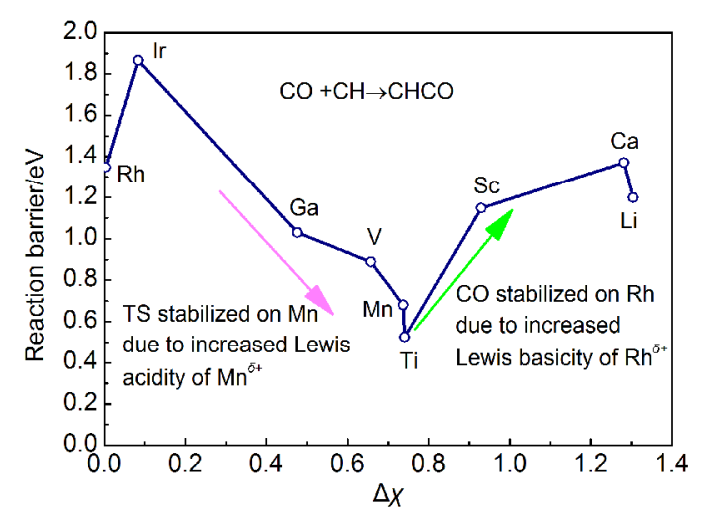

图 $6 \mathrm{DFT}$ 计算出的 $\mathrm{Rh} / \mathrm{M}$ 粒子上 $\mathrm{CO}+\mathrm{CH} \rightarrow \mathrm{CHCO}$ 反应 能垒与 $\mathrm{Rh}$ 和各助剂 $M$ 电负性差值 $(\Delta \chi)$ 的关系 ${ }^{65}$

Fig.6 DFT calculated activation barriers of $\mathrm{CO}+\mathrm{CH} \rightarrow$ $\mathrm{CHCO}$ on the $\mathrm{Rh} / \mathrm{M}$ particles as a function of electronegativity differences $(\Delta \chi)$ between $\mathrm{Rh}$ and various promoters ${ }^{65}$

TS: transition state

$\mathrm{CO}$ 稳定存在于其上. 当 $\Delta \chi$ 超过 0.70 时, $\mathrm{Rh}$ 与 $\mathrm{CO}$ 之 间的键合更强, 则不利于插入反应.

可以看出, $\mathrm{Ti} / \mathrm{Mn}$ 和 $\mathrm{V}$ 与 $\mathrm{Rh}$ 的 $\Delta \chi$ 分别为 0.70 和 0.65 , 因此它们的加入有利于提高 $\mathrm{Rh}$ 基催化剂上 $\mathrm{CO}$ 加氢生成乙醇等 $\mathrm{C}_{2}$ 含氧化合物的选择性和活 性, 这与众多文献结果 ${ }^{11,55-60}$ 一致. 结果还显示, 对于 三元催化剂体系, 助剂与 $\mathrm{Rh}$ 的 $\Delta \chi=0.60-0.90$ 时, 对 反应较为有利, 如 $\mathrm{Rh}-\mathrm{V}-\mathrm{La} / \mathrm{SiO}_{2}$ 体系. ${ }^{83}$ 因此, $\Delta \chi$ 值可 为选择适宜 $\mathrm{Rh}$ 基催化剂助剂提供指导, 但仍需更多 的理论和实验证实.

Ichikawa $^{84,85}$ 曾考察了载体酸碱性对其负载的 $\mathrm{Rh}$ 催化剂性能的影响, Prieto 等 ${ }^{86}$ 则系统深入研究 了助剂氧化物 $\mathrm{MO}_{x}$ 的酸碱性(拉/给电子)对 $\mathrm{Rh} / \mathrm{Al}_{2} \mathrm{O}_{3}$ 催化剂上 $\mathrm{CO}$ 加氢活性和产物选择性的关系. 考虑 到载体织构性质的均一性, 同时最大化助剂- Rh 接 触面积, 在制备过程中先将载体上单层覆盖金属氧 化物 $\mathrm{M} @ \mathrm{Al}_{2} \mathrm{O}_{3}$, 然后浸渍 $\mathrm{Rh}$ 的前驱体而制得 $\mathrm{Rh} /$ $\mathrm{M} @ \mathrm{Al}_{2} \mathrm{O}_{3}$ 催化剂. 该系列 $\mathrm{M} @ \mathrm{Al}_{2} \mathrm{O}_{3}$ 在较宽范围内具 有不同的拉电子/给电子(Lewis 酸/碱性) 性质: $\mathrm{VO}_{x}$ 、 $\mathrm{NbO}_{x} 、 \mathrm{TaO}_{x}$ 酸性较强, $\mathrm{FeO}_{x} 、 \mathrm{PrO}_{x} 、 \mathrm{NdO}_{x}$ 和 $\mathrm{SmO}_{x}$ 碱性 较强, 而 $\mathrm{YO}_{x}$ 和 $\mathrm{TiO}_{x}$ 酸碱性适中. 结果发现, 这些性 质与催化剂的活性和选择性存在很好的关联, 如图 7 所示, 其中 $\Phi=C_{\text {ins }} / C_{\text {dis, }}$, 即产物中 $\mathrm{CO}$ 插入与解离数 目的比值, 为产物选择性系数. 对于还原的和反应 时的催化剂, 当其助剂氧化物 (如 $\mathrm{TaO}_{x}$ ) 具有拉电子 性质(酸性)时, $\mathrm{Rh}$ 上桥式吸附 $\mathrm{CO}$ 的比例更高, 平板 状 $\mathrm{Rh}$ 纳米粒子的电子反馈能力更高, 有利于 $\mathrm{CO}$ 解 


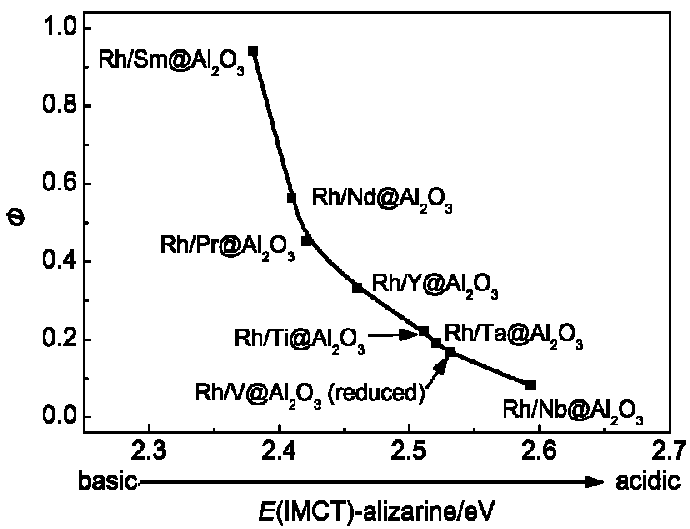

图 $7 \mathrm{Rh} / \mathrm{M} @ \mathrm{Al}_{2} \mathrm{O}_{3}$ 催化剂上 $\mathrm{CO}$ 加氢产物选择性 参数 $(\Phi)$ 与助剂 $\mathrm{MO}_{x}$ 电子性质的关系 ${ }^{86}$

Fig.7 Evolution of the selectivity parameter $(\Phi)$ with the electronic properties of the MO $_{x}$ promoter phase ${ }^{86}$ $E(\mathrm{IMCT})$ : the lowest-energy intramolecular charge-transfer energy, using adsorbed alizarine as descriptor; prereduced for $\mathrm{VO}_{x}$ and calcined for the remaining $\mathrm{MO}_{x}$

离, 因而催化剂活性和烃类选择性更高; 相反地, 而 当助剂氧化物 (如 $\mathrm{SmO}_{x}$ ) 具有给电子性质(碱性)时, $\mathrm{Rh}$ 位的电子反馈能力较低, 则导致更高的非解离 $\mathrm{CO}$ 的插入活性, 因而含氧化合物选择性更高. 这与 Ichikawa $^{84,85}$ 的很类似.

上述结果提示我们在选择 $\mathrm{Rh}$ 基催化剂助剂时, 可从金属的电负性和相应氧化物的酸碱性等基本 特性入手, 具有一定的指导意义.

\section{4 结论与展望}

助剂、金属和载体间相互作用的程度决定了催 化剂的性能, 金属-过渡金属助剂间紧密接触所产生 的界面处往往是生成 $\mathrm{C}_{2}$ 含氧化合物的中心, 因此要 优化助剂-金属间相互作用, 提高界面面积, 其中助 剂含量和制备方法是重要的调节手段. Fe 主要通过 与 $\mathrm{Rh}$ 的紧密接触创造新的活化 $\mathrm{CO}$ 及其插入活性 位, 从而提高 $\mathrm{CO}$ 加氢生成含氧化合物, 同时增加了 催化剂加氢活性, 因此显著提高了乙醇和甲醇的生 成活性和选择性; $\mathrm{Mn}$ 通过与 $\mathrm{Rh}$ 的相互作用, 促进 $\mathrm{CO}$ 的解离和插入, 可总体提高 $\mathrm{C}_{2}$ 含氧化合物的活 性和选择性; $\mathrm{Li}$ 通过覆盖 $\mathrm{Rh}$ 表面或电子作用以抑 制催化剂的加氢能力, 从而大大提高了 $\mathrm{C}_{2}$ 含氧化合 物的选择性, 尤其是加氢程度最低的乙酸, 但一般 会导致催化剂活性有所下降. 总之, Mn、Li、Fe 是合 成气制 $\mathrm{C}_{2}$ 含氧化合物最有效的助剂之一, 它们的作 用本质必须与催化剂体系(组分和含量)、制备、活化 和反应条件等具体情形结合起来, 才能得到正确的
解释. 另外, 单组分促进的 $\mathrm{Rh}$ 基催化剂性能很难达 到理想的水平, 但助剂的作用具有叠合性, 有些情 况下, 还具有协同效应. 因此, 同时添加 $\mathrm{Mn} 、 \mathrm{Li} 、 \mathrm{Fe}$ 等多种助剂, 综合它们的优点, 才有可能制得到性 能较好的 $\mathrm{Rh}$ 基催化剂. 就目前现状而言, 助剂的篎 选意义不大, 最主要的是寻找合适的制备方法和结 构特殊的载体材料, 优化这些常用助剂、载体与 $\mathrm{Rh}$ 的相互作用, 最大限度地提高 $\mathrm{Rh}$ 的催化效率, 将 $\mathrm{Rh}$ 含量降低至 $0.1 \%$ 左右, 才能有力推动该过程的工业 化进程. 另一方面, 非 $\mathrm{Rh}$ 催化剂的开发也是实现合 成气制乙醇等 $\mathrm{C}_{2}$ 含氧化合物的重要选项, 但任务同 样艰巨.

\section{References}

(1) Subramani, V.; Gangwal, S. K. Energy Fuels 2008, 22 (2), 814

(2) Spivey, J. J.; Egbebi, A. Chem. Soc. Rev. 2007, 36 (9), 1514.

(3) Chen, W. M.; Ding, Y. J.; Xue, F.; Song, X. G. Zhu, H. J.; Lv, Y. Chem. Ind. Eng. Prog. 2014, 33 (7), 1753. [陈维苗, 丁云杰, 薛飞, 宋宪根, 朱何俊, 吕 元 . 化工进展, 2014, 33 (7), 1753.]

(4) Bhasin, M. M.; Charleston, W. V. Verfahren Zur Herstellung Von Athanol Aus Synthese gas. DE2503204, 1975.

(5) Arimitu, S.; Tanaka, K.; Saito, T. The Research Association for $\mathrm{C}_{1}$ Chemistry. Progress in $\mathrm{C}_{1}$ Chemistry in Japan. Elsevier, Amsterdam, 1989; pp 1-240.

(6) Yu, J.; Mao, D. S.; Guo, S. Q.; Han, L. P.; Lu, G. Z. Acta Phys. -Chim. Sin. 2012, 28 (3), 667. [俞 俊, 毛东森, 郭强 胜, 韩璐蓬, 卢冠忠. 物理化学学报, 2012, 28 (3), 667.] doi: 10.3866/PKU.WHXB201112221

(7) Luo, H. Y.; Lin, P. Z.; Xie, S. B.; Zhou, H. W.; Xu, C. H.; Huang, S. Y.; Lin, L. W.; Liang, D. B.; Yin, P. L.; Xin, Q. J. Mol. Catal. A 1997, 122, 115.

(8) Yin, H. M.; Ding, Y. J.; Luo, H. Y.; Zhu, H. J.; He, D. P.; Xiong, J. M.; Lin, L. W. Appl. Catal. A 2003, 243 (1), 155.

(9) Chen, W. M.; Ding, Y. J.; Jiang, D. H.; Jiao, G. P.; Zhu, H. J.; Pan, Z. D.; Luo, H. Y. Chin. J. Catal. 2006, 27 (11), 999. [陈维 苗, 丁云杰, 江大好, 焦桂萍, 朱何俊, 潘振栋, 罗洪原. 催化学 报, 2006, 27 (11), 999.]

(10) Chen, W. M.; Ding, Y. J.; Luo, H. Y.; Yan, L.; Wang, T.; Pan, Z. D.; Zhu, H. J. Chin. J. Appl. Chem. 2005, 22 (5), 470. [陈维 苗, 丁云杰, 罗洪原, 严 丽, 王 涛, 潘振栋, 朱何俊. 应用化 学, 2005, $22(5), 470$.

(11) Chen, W. M.; Ding, Y. J.; Jiang, D. H.; Pan, Z. D.; Luo, H. Y. J. Nat. Gas. Chem. 2005, 14, 199.

(12) Chen, W. M.; Ding, Y. J.; Jiang, D. H.; Pan, Z. D.; Luo, H. Y. Catal. Lett. 2005, 104 (3-4), 177. doi: 10.1007/s10562-0057948-6

(13) Jiang, D. H.; Ding, Y. J.; Pan, Z. D.; Chen, W. M.; Luo, H. Y. Catal. Lett. 2008, 121 (3-4), 241. doi: 10.1007/s10562-0079322-3 
(14) Jiang, D. H.; Ding, Y. J.; Lv, Y. Chin. J. Catal. 2009, 30 (7), 697. [江大好, 丁云杰, 吕 元. 催化学报, 2009, 30 (7), 697.]

(15) Li, J. W.; Ding, Y. J.; Lin, R. H.; Gong, L. F.; Song, X. G.; Chen, W. M.; Wang, T.; Luo, H. Y. Chin. J. Catal. 2010, 31 (3), 365. [李经伟, 丁云杰, 林荣和, 龚否峰, 宋宪根, 陈维苗, 王 涛, 罗 洪原. 催化学报, 2010,31 (3), 365.]

(16) Chen, W. M.; Ding, Y. J.; Song, X. G.; Wang, T.; Luo, H. Y. Appl. Catal. A 2011, 407, 231.

(17) Chen, W. M.; Ding, Y. J.; Song, X. G. Chin. J. Catal. 2012, 33 (6), 1007. [陈维苗, 丁云杰, 宋宪根. 催化学报, 2012, 33 (6), 1007.]

(18) Song, X. G.; Ding, Y. J.; Chen, W. M.; Dong, W. D.; Pei, Y. P.; Zang, J.; Yan, L.; Lv, Y. Catal. Commun. 2012, 19, 100. doi: 10.1016/j.catcom.2011.12.015

(19) Li, C.; Liu, J.; Gao, W.; Zhao, Y.; Wei, M. Catal. Lett. 2013, 143, 1247. doi: 10.1007/s10562-013-1100-9

(20) Kim, M. J.; Chae, H. J.; Ha, K. S.; Jeong, K. E.; Kim, C. U.; Jeong, S. Y.; Kim, T. W. J. Porous Mater. 2014, 21, 365. doi: 10.1007/s10934-014-9782-y

(21) Zhu, L. J.; Guo, W. W.; Wang, H. X. Adv. Mater. Res. 2014, $864-867,442$

(22) Ichikawa, M.; Fukushima, T. J. Chem. Soc. Chem. Commun. 1985, No. 6, 321

(23) Chuang, S. S. C.; Stevens, R. W., Jr.; Khatri, R. Top. Catal. 2005, 32 (3-4), 225. doi: 10.1007/s11244-005-2897-2

(24) Bahasin, M. M.; Bartley, W. J.; Ellgen, P. C.; Wilson, T. P. J. Catal. 1978, 54, 120. doi: 10.1016/0021-9517(78)90035-0

(25) Ellgen, P. C.; Bartley, W. J.; Bhasin, M. M.; Wilson, T. P. $A d v$. Chem. Ser. Am. Chem. Soc. 1979, 178, 147. doi: 10.1021/ advances

(26) Ichikawa, M.; Fukushima, T.; Shikakura, K.; Tominaga, T. The Role of $\mathrm{Fe}, \mathrm{Mn}$, and Ti-Oxide Additives Modifying $\mathrm{C}_{2}-$ Oxygenate Formation Catalyzed by $\mathrm{SiO}_{2}$-Supported RhContaining Catalysts in a CO- $\mathrm{H}_{2}$ Conversion. In Prceceedings of 8th International Congress on Catalysis; Berlin (West), July 2-6, 1984; Verlag Cheme: Weinheim, 1984; Vol. V, pp 85-111.

(27) Fukushima, T.; Arakawa, H.; Ichikawa, M. J. Phys. Chem. 1985, 89, 4440. doi: 10.1021/j100267a009

(28) Ichikawa, M.; Fukushima, T. J. Phys. Chem. 1985, 89, 1564. doi: 10.1021/j100255a003

(29) Burch, R.; Petch, M. I. Appl. Catal. A 1992, 88, 39. doi: 10.1016/ 0926-860X(92)80195-I

(30) Burch, R.; Petch, M. I. Appl. Catal. A 1992, 88, 77. doi: 10.1016/ 0926-860X(92)80197-K

(31) Burch, R.; Petch, M. I. Appl. Catal. A 1992, 88, 61. doi: 10.1016/ 0926-860X(92)80196-J

(32) Gao, J.; Mo, X.; Goodwin, J. G., Jr. J. Catal. 2009, 268, 142. doi: 10.1016/j.jcat.2009.09.012

(33) Guglielminotti, E.; Pinna, F.; Rigoni, M.; Strukul, G.; Zanderighi, L. J. Mol. Catal. A 1995, 103, 105. doi: 10.1016/ 1381-1169(95)00119-0
(34) Ichikawa, M.; Fukushima, T.; Yokoyama, T.; Kosugi, N.; Kuroda, H. J. Phys. Chem. 1986, 90, 1222. doi: 10.1021/ j100398a003

(35) Fukuoka, A.; Ichikawa, M.; Hriljac, J. A.; Shriver, D. F. Inorg. Chem. 1987, 26, 3643. doi: 10.1021/ic00269a001

(36) Fukuoka, A.; Kimura, T.; Kosugi, N.; Kuroda, H.; Minai, Y.; Sakai, Y.; Tominaga, T.; Ichikawa, M. J. Catal. 1990, 126, 434. doi: 10.1016/0021-9517(90)90010-H

(37) Boffa, A.; Lin, C.; Bell, A. T.; Somorjai, G. A. J. Catal. 1994, 149, 149. doi: 10.1006/jcat.1994.1280

(38) Haider, M. A.; Gogate, M. R.; Davis, R. J. J. Catal. 2009, 261 (1), 9. doi: 10.1016/j.jcat.2008.10.013

(39) Choi, Y.; Liu, P. J. Am. Chem. Soc. 2009, 131, 13054. doi: 10.1021/ja903013x

(40) Niemantsverdriet, J. W.; van der Kraan, A. M.; Delgass, W. N. J. Catal. 1984, 89, 138. doi: 10.1016/0021-9517(84)90288-4

(41) Niemantsverdriet, J. W.; van der Kraan, A. M.; Delgass, W. N. J. Phys. Chem. 1983, 87, 1292. doi: 10.1021/j100231a005

(42) Schuenemann, V.; Trevino, H.; Sachtler, W. M. H.; Fogash, K.; Dumesic, J. A. J. Phys. Chem. 1995, 99, 1317. doi: 10.1021/ j100004a036

(43) Schunemann, V.; Trevino, H.; Lei, G. D.; Tomczak, D. C.; Sachtler, W. M. H.; Fogash, K.; Dumesic, J. A. J. Catal. 1995, 153, 144. doi: 10.1006/jcat.1995.1116

(44) Gogate, M. R.; Davis, R. J. ChemCatChem 2009, 1 (2), 295. doi: $10.1002 /$ cctc.v1:2

(45) Zakubaeva, G. D.; Beketaeva, L. A.; Uvaliev, T. Y.; Khiystov, A. S.; Litvyakova, E. N. React. Kinet. Catal. Lett. 1985, 28 (2), 425. doi: 10.1007/BF02062976

(46) Burch, R.; Hayes, M. J. J. Catal. 1997, 165, 249. doi: 10.1006/ jcat.1997.1482

(47) Qin, S. D.; Zhang, C. H.; Xu, J.; Wu, B. S.; Xiang, H. W.; Li, Y. W. Chin. J. Catal. 2010, 31 (9), 1132. [秦绍东, 张成华, 许 健, 吴宝山, 相宏伟, 李永旺. 催化学报, 2010, 31 (9), 1132.]

(48) Chen, G. C.; Guo, C.; Huang, Z.; Yuan, G. Chem. Eng. Res. Des. 2011, 89 (3A), 249.

(49) Wang, J.; Zhang, Q.; Wang, Y. Catal. Today 2011, 171, 257. doi: 10.1016/j.cattod.2011.03.023

(50) Yu, J.; Mao, D.; Han, L.; Guo, Q.; Lu, G. Fuel Process. Technol. 2013, 112, 100. doi: 10.1016/j.fuproc.2013.03.004

(51) Yu, J.; Mao, D.; Han, L.; Guo, Q.; Lu, G. Catal. Commun. 2012, 27, 1. doi: 10.1016/j.catcom.2012.06.010

(52) Yu, J.; Mao, D.; Han, L.; Guo, Q.; Lu, G. J. Ind. Eng. Chem. 2013, 19, 806. doi: 10.1016/j.jiec.2012.10.021

(53) Mo, X.; Gao, J.; Umnajkaseam, N.; Goodwin, J. G. J. Catal. 2009, 267, 167. doi: 10.1016/j.jcat.2009.08.007

(54) Sachtler, W. M. H.; Ichikawa, M. J. Phys. Chem. 1986, 90, 4752. doi: 10.1021/j100411a009

(55) Shriver, D. F. Activation of Carbon Monoxide by Carbon and Oxygen Coordination Lewis Acid and Proton Induced Reduction of Carbon Monoxide. In ACS Symposium Series; 
ACS Publications: 1981; Vol. 152, Chapter 1, pp 1-8. http:// pubs.acs.org/doi/abs/10.1021/bk-1981-0152. ch001. doi: 10.1021/symposium

(56) Wilson, T. P.; Kasai, P. H.; Ellgen, P. C. J. Catal. 1981, 69 , 193. doi: 10.1016/0021-9517(81)90141-X

(57) Van der Berg, F. G. A.; Glezer, J. H. E.; Sachtler, W. M. H. J. Catal. 1985, 93, 340. doi: 10.1016/0021-9517(85)90181-2

(58) Reys, P.; Concha, I.; Pecchi, G.; Fierro, J. L. G. J. Mol. Catal. A 1998, 129, 269. doi: 10.1016/S1381-1169(97)00186-6

(59) Lowenthal, E. E.; Allard, L. F.; Te, M.; Foley, H. C. J. Mol. Catal. A 1995, 100, 129. doi: 10.1016/1381-1169(95)00177-8

(60) Wang, Y.; Luo, H. Y.; Liang, D. B.; Bao, X. H. J. Catal. 2000, 196, 46. doi: 10.1006/jeat.2000.3026

(61) Ojeda, M.; Granados, M. L.; Rojas, S.; Terreros, P.; GarciaGarcia, F. J.; Fierro, J. L. G. Appl. Catal. A 2004, 261 (1), 47.

(62) Trevino, H.; Lei, G. D.; Sachtler, W. M. H. J. Catal. 1995, 154, 245. doi: $10.1006 /$ jcat. 1995.1166

(63) Trevino, H.; Hyeon, T.; Sachtler, W. M. H. J. Catal. 1997, 170, 236. doi: $10.1006 /$ jcat.1997.1756

(64) Chen, G. C.; Zhang, X. H.; Guo, C. Y.; Yuan, G. Q. C. R. Chim. 2010, 13, 1384. doi: 10.1016/j.crci.2010.03.025

(65) Mei, D.; Rousseau, R.; Kathmann, S. M.; Glezakou, V. A.; Engelhard, M. H.; Jiang, W.; Wang, C.; Gerber, M. A.; White, J. F.; Stevens, D. J. J. Catal. 2010, 271, 325. doi: 10.1016/j. jcat.2010.02.020

(66) Ma, X. F.; Su, H. Y.; Deng, H. Q.; Li, W. X. Catal. Today 2010, $160(1), 228$.

(67) Li, F.; Jiang, D. E.; Zeng, X. C.; Chen, Z. Nanoscale 2012, 4, 1123. doi: $10.1039 / \mathrm{c} 1 \mathrm{nr} 11121 \mathrm{c}$

(68) Huang, L.; Deng, W. H.; Guo, E.; Chung, P. W.; Chen, S.; Trewyn, B. G.; Brown, R. C.; Lin, V. S. Y. ChemCatChem 2012, 4, 674. doi: $10.1002 /$ ctc.v4.5

(69) Liu, J. J.; Guo, Z.; Childers, D.; Regalbuto, J. R.; Marshall, C. L.; Klie, R. F.; Miller, J. T.; Meyer, R. J. ChemCatChem 2013, 5, 3665. doi: 10.1002/cctc.201300479

(70) Liu, J. J.; Guo, Z.; Childers, D.; Schweitzer, N.; Marshall, C. L.; Klie, R. F.; Miller, J. T.; Meyer, R. J. J. Catal. 2014, 313,
149. doi: $10.1016 /$ j.jcat.2014.03.002

(71) Wang, Y.; Song, Z.; Ma, D.; Luo, H. Y.; Liang, D. B.; Bao, X. H. J. Mol. Catal. A 1999, 149, 51. doi: 10.1016/S1381-1169(99) 00181-8

(72) Bao, H. L.; Sun, X. P.; Jiang, Z.; Huang, Y. Y.; Wang, J. Q. Chin. J. Catal. 2014, 35 (8), 1418. [鲍洪亮, 孙雪平, 姜 政, 黄宇 营, 王建强. 催化学报, 2014, 35 (8), 1418.] doi: 10.1016/S18722067(14)60081-4

(73) Kesraoui, S.; Oukaci, R.; Blackmond, D. G. J. Catal. 1987, 105, 432. doi: 10.1016/0021-9517(87)90071-6

(74) Blackmond, D. G.; Williams, J. A.; Kesraoui, S.; Blazewick, D. S. J. Catal. 1986, 101, 496. doi: 10.1016/0021-9517(86)90276-9

(75) Gallaher, G. R.; Goodwin, J. G.; Huang, C. S.; Houalla, M. J. Catal. 1993, 140, 453. doi: 10.1006/jcat. 1993.1098

(76) Chuang, S. C.; Goodwin, J. G.; Wender, I. J. Catal. 1985, 95 , 435. doi: 10.1016/0021-9517(85)90121-6

(77) Chuang, S. C.; Goodwin, J. G.; Wender, I. J. Catal. 1985, 92, 416. doi: 10.1016/0021-9517(85)90275-1

(78) Egbebi, A.; Schwartz, V.; Overbury, S. H.; Spivey, J. J. Catal. Today 2010, 149, 91. doi: 10.1016/j.cattod.2009.07.104

(79) Schwartz, V.; Campos, A.; Egbebi, A.; Spivey, J. J.; Overbury, S. H. ACS Catal. 2011, 1, 1298. doi: 10.1021/cs200281g

(80) Kusama, H.; Okabe, K.; Sayama, K.; Arakawa, H. Catal. Today 1996, 28, 261. doi: 10.1016/0920-5861(95)00246-4

(81) Ngo, H.; Liu, Y.; Murata, K. React. Kinet. Mech. Catal. 2011, 102 (2), 425. doi: 10.1007/s11144-010-0263-2

(82) Hargis, D. C.; Dubeck, M. Ethanol from Methanol and Synthesis Gas. US4370507, 1983.

(83) Gao, J.; Mo, X.; Chien, A. C. Y.; Goodwin, J. G., Jr. J. Catal. 2009, 262, 119. doi: 10.1016/j.jcat.2008.12.006

(84) Ichikawa, M. Bull. Chem. Soc. Jpn. 1978, 51, 2268. doi: 10.1246/bcsj.51.2268

(85) Ichikawa, M. Bull. Chem. Soc. Jpn. 1978, 51, 2273. doi: 10.1246/bcsj. 51.2273

(86) Prieto, G.; Concepcion, P.; Martinez, A.; Mendoza, E. J. Catal. 2011, 280, 274. doi: 10.1016/j.jcat.2011.03.025 